PHYSICAL REVIEW D 85, 089908(E) (2012)

\title{
Publisher's Note: Supersymmetric double field theory: A stringy reformulation of supergravity [Phys. Rev. D 85, 081501(R) (2012)]
}

Imtak Jeon (전임탁), Kanghoon Lee (이강훈), and Jeong-Hyuck Park (박정혁)

(Received 16 April 2012; published 30 April 2012)

DOI: 10.1103/PhysRevD.85.089908

PACS numbers: 04.60.Cf, 04.65.+e, 99.10.Fg

This paper was published online on 2 April 2012 with an incorrect author affiliation. Jeong-Hyuck Park's affiliation should read as "2Department of Physics, Sogang University, Seoul 121-742, Korea”. The author's affiliation has been corrected as of 17 April 2012. The affiliation is correct in the printed version of the journal. 